\title{
DEPROJECTION OF GALAXIES: HOW MUCH CAN BE LEARNED?
}

\author{
George B. Rybicki \\ Harvard-Smithsonian Center for Astrophysics \\ 60 Garden Street \\ Cambridge, MA 02138
}

\begin{abstract}
A general discussion, based on the "Fourier Slice Theorem," is given for the problem of deprojecting the observed light distribution of galaxies to obtain their intrinsic three dimensional light distribution or "shape." Several results are obtained: 1) A model-independent deprojection of an axially symmetric galaxy is shown to be possible only if the symmetry axis lies in the plane of the sky. 2) A simple criterion is given to test whether two different galaxies can have the same intrinsic shape, based solely on their observed projections. 3) It is shown that a homogeneous class of galaxies can be deprojected using a sufficiently large number of projections of random perspective.
\end{abstract}

\section{THE FOURIER SLICE THEOREM}

The intrinsic shape of a galaxy, defined by its light distribution in space $\epsilon(x, y, z)$, determines the brightness projection, $I(x, y)=\int \epsilon(x, y, z) d z$, onto the $x-y$ plane of the sky. An important question is to what extent this projection can be used to determine the intrinsic shape of the galaxy, what is usually called the deprojection problem. In principle other kinds of information (such as velocity data) might help to determine the intrinsic shape, but the discussion here is confined to the pure deprojection problem, using only projected brightness data.

It is convenient to discuss properties of projections in terms of Fourier transforms. Denoting the three-dimensional Fourier transform of $\epsilon(x, y, z)$ by $\tilde{\epsilon}(u, v, w)$ and the two-dimensional Fourier transform of $I(x, y)$ by $\tilde{I}(u, v)$, it is easily shown that $\tilde{I}(u, v)=\tilde{\epsilon}(u, v, 0)$. The generalization of this result to projections in arbitrary directions is that the values of the two-dimensional Fourier transform of the projection are equal to the values of the three-dimensional Fourier transform of the light distribution along a planar "slice" through the origin and normal to the direction of the projection (Bracewell and Riddle 1967; Crowther et al. 1970). There are several immediate consequences of this "Fourier slice theorem":

1) Axially symmetric Galaxies. Since the intrinsic shape of an axially symmetric galaxy and its projection are both determined by functions of two variables, it is tempting to believe that an inversion formula might be found for this case. To investigate this, one notes that the one observed projection yields information in the Fourier slice normal to the line of sight. Now the axial symmetry implies that exactly the same view would be seen at another perspective related to the original 
one by a simple rotation about the symmetry axis. Thus we can rotate the original Fourier slice about the symmetry axis, yielding information about other parts of the Fourier domain. If the symmetry axis lies in the plane of the sky, the entire Fourier space is covered in this rotation. However, if the symmetry axis is at an angle $\theta>0$ to the plane of the sky, then this rotation of the Fourier slice leaves a "cone of ignorance," of half-angle $\theta$, such that Fourier components within this cone remain completely unknown. Therefore if $\theta>0$ the inversion problem for axially symmetric galaxies has no solution.

2) A Test of Identity. Consider two projections of a single galaxy. Using the Fourier slice theorem, one obtains the values along the two slices of the Fourier space normal to the two directions of projection. Since these two planes intersect in a line, the complex values along this line must be identical for the two projections (Crowther et al. 1970). This fact can be used as a test to determine if two different galaxies seen in projection can have the same intrinsic shape. After Fourier transforming the two projections, one looks for a line through the origin of each of these Fourier planes (in general at different angles) such that the Fourier values are identical along them. If such lines are found it is at least possible for the intrinsic shapes to be indentical. Otherwise, the possibility of having the same intrinsic shape is ruled out.

In practice the relative scaling of two projections might be difficult to determine, so it is appropriate to include the possibility of a simple rescaling the data by a uniform stretching or shinking in order to match the two lines of Fourier data. The identity of two functions is such a strong condition that it is not substantially weakened by allowing such rescalings.

3) Deprojection of a Homogeneous Class of Galaxies. Suppose there existed a homogeneous class of galaxies, all with the same intrinsic shape. From the preceding discussion, the Fourier transforms of any two projections would have a line of values in common (to within a simple rescaling). The corresponding Fourier slices could be fitted together along that line, but the angle between the planes would be undetermined. Now taking a third projection, one could locate the lines of common values with each of the first two projections, and this third plane could be fitted in, yielding a rigid structure for the three planes. Further planes could similarly be inserted, and, if the observed projections were sufficiently numerous and varied, eventually the Fourier space would be filled in enough for an inversion to be performed.

\section{REFERENCES}

Bracewell, R. N., and Riddle, A. C. 1967, Astrophys. J., 150, 427.

Crowther, R. A., DeRosier, D. J., and Klug, A. 1970, Proc. Roy. Soc. Lond. A. 317, 319. 